\section{Recurrent respiratory obstruction from a mediastinal bronchogenic cyst}

\author{
S R D Johnston, A Adam, D J Allison, \\ $P$ Smith, $P$ W Ind
}

\begin{abstract}
A large mediastinal bronchogenic cyst presented acutely with paroxysmal atrial fibrillation and severe airflow obstruction. The patient had experienced identical symptoms on two other occasions over the previous 24 years. These had been previously misdiagnosed as due to a mediastinal lymphoma. Percutaneous extrapleural aspiration successfully decompressed the cyst with substantial improvement in lung function. Recurrent swelling of the cyst occurred that could not be relieved surgically. After repeat aspiration percutaneous instillation of bleomycin and alcohol has been used to prevent further increase in the size of the cyst.
\end{abstract}

Bronchogenic cysts are congenital abnormalities resulting from embryological budding of the bronchial tree that often arise near the carina. ${ }^{1}$ In adults they are often incidental findings on a chest radiograph but they may present with respiratory distress due to major airways compression. Thus, despite their benign nature, surgical excision is often undertaken to confirm the diagnosis and prevent future complications. We report a case where a mediastinal bronchogenic cyst presented with recurrent airflow obstruction, in which surgical removal was not possible because of previous radiotherapy.

Division of

Respiratory Medicine

S R D Johnston

$P$ W Ind

Department of

Radiology

A Adam

D J Allison

Department of

Surgery

P Smith

Hammersmith

Hospital, London

W12 0HS

Reprint requests to:

Dr P W Ind

Accepted 23 January 1992

\section{Case report}

A 44 year old woman presented with a four day history of palpitations, retrosternal chest pain and a dry cough. Over two days she became increasingly breathless on minimal exertion and lying flat, and found difficulty in swallowing solid food. On examination she was in atrial fibrillation $(180 / \mathrm{min})$, but sinus rhythm returned with coughing. Some wheeze over the anterior chest was heard, but otherwise examination showed nothing abnormal.

Her past medical history revealed two similar episodes. In 1967 during her second pregnancy she developed intermittent retrosternal chest pain. A rounded mediastinal opacity noted on the chest radiograph resolved after delivery, and no further investigations were undertaken. In 1978 she presented with pleuritic chest pain and breathlessness relieved by sitting forward. Chest radiography showed a right hilar mass compressing the trachea and left main bronchus. She was hypoxic (oxygen tension $7 \cdot 0 \mathrm{kPa}$ ) and had a raised erythrocyte sedimentation rate ( $84 \mathrm{~mm}$ in one hour). She deteriorated over 24 hours and pulmonary angiography showed complete occlusion of the right pulmonary artery by the mediastinal mass. A clinical diagnosis of a rapidly enlarging lymphoma was made and treatment with combination chemotherapy ("CHOP"-cyclophosphamide, hydroxyurea, vincristine, and prednisolone) was given. There was dramatic improvement in her symptoms over 36 hours with reduction in the size of the mass on the chest radiograph. Subsequent mediastinoscopy, undertaken to establish a tissue diagnosis, showed only necrotic tissue. She completed four cycles of chemotherapy as well as mediastinal radiotherapy, and remained well for 12 years.

Investigations performed when she was admitted on this occasion showed a normal full blood count and biochemical profile but a raised erythrocyte sedimentation rate $64 \mathrm{~mm}$ in one hour). Blood gas tensions while she breathed air were normal. Chest radiography suggested a widened carina, a right hilar shadow, and a narrowed left main bronchus. Spirometry confirmed airflow obstruction with the forced expiratory volume in one second $\left(\mathrm{FEV}_{1}\right)$ reduced to $45 \%$ predicted, $\mathrm{FEV}_{1} /$ forced vital capacity

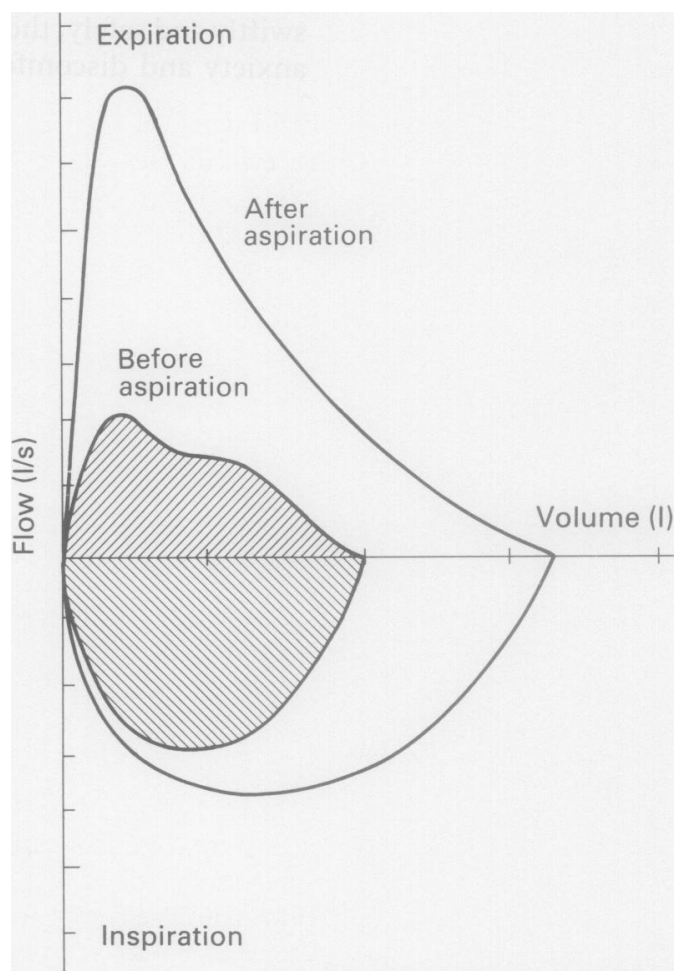

Figure 1 Flow-volume loops before and after aspiration of the bronchogenic cyst. 
Figure 2 Computed tomogram of the mediastinum showing a well defined $8 \times 8 \mathrm{~cm}$ bronchogenic cyst compressing the main bronchi at the level of the carina.

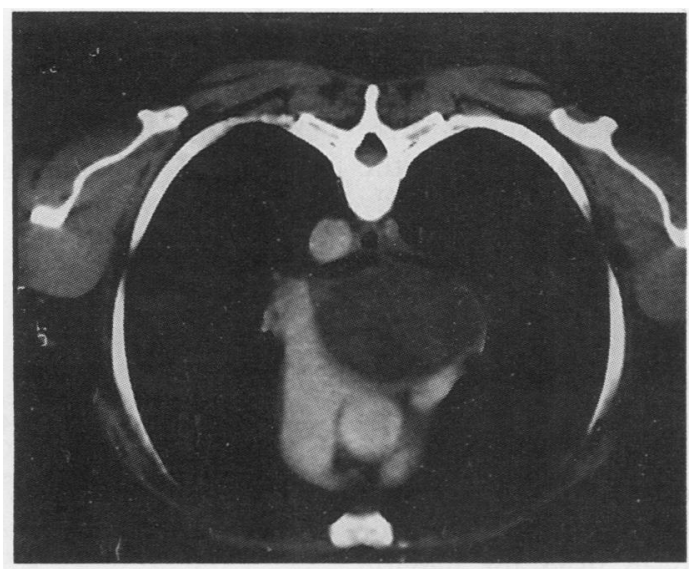

(FVC) ratio $64 \%$, and reduced peak expiratory flow (PEF) $180 \mathrm{l} / \mathrm{min}$ (42\% predicted). The flow-volume loop (fig 1) showed a reduced expiratory loop with a low $\mathrm{E}_{50}: \mathrm{E}_{150}$ ratio of 0.41 , consistent with significant intrathoracic major airways obstruction. Computed tomography of the chest showed a well defined mass, $8 \times 8 \mathrm{~cm}$, of homogeneous attenuation (17 Hounsfield units) within the mediastinum, compressing both main bronchi and displacing adjacent structures, including the left atrium and oesophagus (fig 2). The radiological findings were diagnostic of a bronchogenic cyst and bronchoscopy was not performed because of her severe airways obstruction.

Surgery was more or less contraindicated because of her previous radiotherapy and her obesity (she was $124 \mathrm{~kg}$ ). In view of her progressive obstructive symptoms aspiration of the cyst was performed to relieve major airways obstruction. A percutaneous extrapleural approach, guided by computed tomography, was used ${ }^{2}$ and $110 \mathrm{ml}$ of "dirty" brown fluid removed. Cytological analysis of the fluid showed no malignant cells but clumps of ciliated columnar epithelium with sheets of degenerate macrophages. Cultures of the cyst fluid were sterile and there was no evidence of recent haemorrhage. Further scans showed the cyst to be multilocular but attempts to aspirate the various compartments were unsuccessful because of the thick fibrous wall, presumably resulting from previous radiotherapy. After aspiration there was considerable improvement in her breathlessness. Repeat spirometry showed $\mathrm{FEV}_{1}$ to be $103 \%$ predicted, the $\mathrm{FEV}_{1}$ / FVC ratio normal, and PEF $435 \mathrm{l} / \mathrm{min}$ (116\% predicted), with restoration of a normal flowvolume loop (fig 1). After aspiration computed tomography showed a small residual thick walled cyst with patent major airways.

She remained well for only a few weeks before presenting again with acute breathlessness, wheeze, and retrosternal chest pain. PEF was reduced to $1101 / \mathrm{min}$ ( $35 \%$ predicted) and the chest radiograph again showed substantial compression of the left main bronchus. Further computed tomography confirmed recurrent swelling of the cyst. She deteriorated over 24 hours and emergency cardiothoracic surgery was undertaken to decompress the cyst. Extensive mediastinal fibrosis was found and it was impossible to define the anatomical limitations of the cyst. Postoperatively her symptoms were partially relieved, but a month later her PEF was only $60 \%$ predicted and further computed tomography showed a large residual cyst. A catheter was therefore inserted into the cyst via a percutaneous extrapleural approach to irrigate and drain it; $250 \mathrm{ml}$ of fluid were removed immediately and a further $230 \mathrm{ml}$ over the next five days. Bleomycin $(40 \mathrm{mg})$ and $100 \%$ alcohol $(40 \mathrm{ml})$ with contrast medium were then instilled into the cyst to induce both a local cytotoxic and a sclerosant effect. After removal of the catheter there was gradual improvement in both symptoms and PEF. Ten months later she remains well with normal lung function; computed tomography shows a small residual cyst that has not increased in size.

\section{Discussion}

Compression of local structures by a bronchogenic cyst may present as breathlessness, atypical chest pain, cough, or-rarely-dysphagia as in this patient. Disturbances of cardiological rhythm are rare and we have found only one previous report. ${ }^{3}$ The initial abnormality on the chest radiograph may often be missed ${ }^{4}$ but computed tomography is diagnostic in almost all cases because of the smooth outline of the cyst and the often low density of the contents, which distinguishes them from solid mediastinal lymph nodes. There has been one previous case report of a bronchogenic cyst that was misdiagnosed and treated as lymphoma, which resulted in considerable fibrosis that prevented complete surgical excision. ${ }^{5}$

This unique case illustrates the long natural history of bronchogenic cysts, which are congenital. Probably during her pregnancy the atypical chest pain and radiological abnormality, which resolved after delivery, represented sudden expansion of the cyst. Expansion of a cyst may occur because of infection, haemorrhage into the cyst, or communication with the main bronchi, allowing air inflation. Changes during pregnancy in the size of a cyst, which then reverts to its previous size, have not been reported previously. The reasons for the recurrent swelling of the cyst in this case over 24 years are not clear.

Surgical management remains the treatment of choice in uncomplicated cases. Surgery is also offered to symptomless individuals because of the potentially life threatening complications of the cyst, as well as the need in some cases for a tissue diagnosis to exclude malignancy. More recently transbronchial or transoesophageal aspiration has been used to establish a diagnosis. ${ }^{6}$ We have previously used a percutaneous extrapleural approach guided by computed tomography to drain a bronchogenic cyst. ${ }^{2}$ This particular technique offers a safer route with a low risk of pneumothorax because the pleura is not traversed. This is the first reported case where this technique has been used acutely to decompress a mediastinal cyst to relieve severe airways obstruction. We documented substantial improvement in spiro-metric values, PEF, and flow-volume loop profiles after aspiration. Such measurements provide a sen- 
sitive analysis of intrathoracic major airways obstruction and should be mandatory investigations in patients with large mediastinal cysts. Abnormalities in these values may indicate appreciable obstruction before the onset of symptoms and thus lead to early intervention.

Cytotoxic chemotherapy is not standard treatment for bronchogenic cysts, though this patient had previously had dramatic relief of airways obstruction when given chemotherapy for a misdiagnosed lymphoma. Cell turnover and secretory activity may have been inhibited to such an extent that the volume of the cyst was reduced below a critical level. Instillation of sclerosant agents has been used for hepatic cysts $^{7}$ and recurrent swelling of thyroid cysts, ${ }^{8}$ but we believe that this is the first time a sclerosant has been instilled percutaneously into a bronchogenic cyst. Although follow up here has been only for six months, this case suggests that instillation of bleomycin and alcohol may have a role in controlling recurrent bronchogenic cysts that cannot be surgically removed.

\section{Addendum}

The latest follow up, 16 months after instillation of bleomycin and alcohol, showed no change in the size of the residual cyst on the computed tomogram and lung function remained normal.

1 Kirwan WO, Walbaum PR, McCormack RJM. Cystic intrathoracic derivatives of the foregut and their complications. Thorax 1973;28:424-8.

2 Whyte MKB, Dollery CT, Adam A, Ind PW. Central bronchogenic cyst: treatment by extrapleural percutabronchogenic cyst: treatment by extraple

3 Watson AJ, Chaudhary BA. Cardiac arrhythmias and abnormal chest roentgenogram. Chest 1987;92:335-6.

4 Di Lorenzo M, Collin P-P, Vaillancourt R, Duranceau A. Bronchogenic cysts. J Pediatric Surgery 1989;24:988-91.

5 Cartmill JA, Hughes CF. Bronchogenic cysts: A persistent dilemma. Aust NZ J Surg 1989;59:253-6.

6 Kuhlman JE, Fishman EK, Wang KP, Zerhouni EA, Siegelman SS. Mediastinal cysts: Diagnosis by CT and needle aspiration. $A J R$ 1988;150:75-8.

7 Kairaluoma MI, Leinonen A, Stahlberg M, Paivansalo M, Kiviniemi H, Siniluoto T. Percutaneous aspiration and alcohol sclerotherapy for symptomatic hepatic cyst. Ann Surg 1989;210:208-15.

8 Edmonds CJ, Tellez $M$. Treatment of thyroid cysts by aspiration and injection of sclerosant. $B M J$ 1987;295:529.

\section{Treatment of desmoid tumours in Gardner's syndrome}

\author{
C G Eden, N M Breach, P Goldstraw
}

\begin{abstract}
A 24 year old woman with Gardner's syndrome developed a massive chest wall desmoid tumour, which required radical excision and prosthetic reconstruction. In view of the local aggresiveness of this tumour and the fact that it does not metastasise a policy of radical surgery when possible is recommended.
\end{abstract}

Desmoid tumours are rare, accounting for $0 \cdot 1 \%$ of all tumours, with an 8:1 female predominance. The overall annual incidence is about $2 \cdot 4 / 1 \mathrm{~m}$ population, but in Gardner's syndrome the incidence ranges from $3.5 \%$ to $17 \%$. Gardner's syndrome, first described in $1953,{ }^{1}$ consists of adenomatous polyps of the gastrointestinal tract, osteomas, epidermoid cysts, lipomas, dental abnormalities, and periampullary carcinomas as well as desmoid tumours. The incidence of the syndrome is 1:14 025 with an equal sex distribution. It appears to be determined by the same gene as familial polyposis coli, Gardner's syndrome representing a more aggressive phenotypic expression. The gene is inherited as an autosomal dominant. We report a case of a desmoid tumour in a young woman with a family history of Gardner's syndrome and discuss treatment.

\section{Case report}

A 24 year old woman presented with a six month history of a rapidly enlarging mass related to a surgical scar beneath her left breast. A large left atrial appendage had been removed through a left posterolateral thoracotomy five years earlier. She had never been pregnant. Her mother had had two desmoid tumours excised from the anterior abdominal wall and had undergone colectomy for carcinoma of the colon complicating polyposis coli. Her maternal grandmother had died of carcinoma of the colon and her brother had died several years earlier after the removal of a $3 \mathrm{~kg}$ tumour from his arm.

Physical examination showed a fit young woman with a $20 \mathrm{~cm}$ diameter firm, fixed mass related to the anterior aspect of a thoracotomy wound and inferior to her breast, displacing it upwards and medially. She also had a $3 \times 4 \mathrm{~cm}$ firm, modle mass behind her right ear which had the clinical characteristics of an epidermoid cyst. Physical examination, including sigmoidoscopy and opthalmoscopy, otherwise showed no abnormalities.

Full blood count, electrolyte concentrations, and results of liver function tests were normal. A barium enema performed earlier that year also showed nothing abnormal. Computed tomography showed a very large soft tissue mass on the left lower anterior chest wall, destroying ribs and invading the left rectus muscle (figure). There was no 